\title{
Preoperative octreotide treatment of acromegaly: long-term results of a randomised controlled trial
}

\author{
S L Fougner ${ }^{1}$, J Bollerslev 2,3, J Svartberg ${ }^{4,5}$, M Øksnes ${ }^{6}$, J Cooper ${ }^{7}$ and S M Carlsen ${ }^{1,8}$ \\ ${ }^{1}$ Department of Endocrinology, Medical Clinic, St Olavs University Hospital, 7006 Trondheim, Norway, ${ }^{2}$ Section of \\ Specialized Endocrinology, Department of Endocrinology, Oslo University Hospital, Rikshospitalet, Oslo, Norway, \\ ${ }^{3}$ Faculty of Medicine, University of Oslo, Oslo, Norway, ${ }^{4}$ Division of Internal Medicine, University Hospital of North \\ Norway, Tromsø, Norway, ${ }^{5}$ Tromsø Endocrine Research Group, Institute of Clinical Medicine, UiT The Arctic \\ University of Norway, Tromsø, Norway, ${ }^{6}$ Department of Medicine, Centre for Clinical Research, Haukeland \\ University Hospital, Bergen, Norway, ${ }^{7}$ Department of Endocrinology, Stavanger University Hospital, Stavanger, \\ Norway and ${ }^{8}$ Unit for Applied Clinical Research, Norwegian University of Science and Technology (NTNU), \\ Trondheim, Norway
}

Correspondence should be addressed to S L Fougner Email stine.fougner@ntnu.no

\section{Abstract}

Objective: Randomised studies have demonstrated a beneficial effect of pre-surgical treatment with somatostatin analogues (SSA) in acromegaly when evaluated early postoperatively. The objective of this study was to evaluate the long-term surgical cure rates.

Methods: Newly diagnosed patients were randomised to direct surgery $(n=30)$ or 6-month pretreatment with octreotide LAR $(n=32)$. The patients were evaluated 1 and 5 years postoperatively. Cure was defined as normal IGF1 levels and by normal IGF1 level combined with nadir $\mathrm{GH}<2 \mathrm{mU} / \mathrm{l}$ in an oral glucose tolerance test, all without additional post-operative treatment. A meta-analysis using the other published randomised study with long-term analyses on preoperative SSA treatment was performed.

Results: The proportion of patients receiving post-operative acromegaly treatment was equal in the two groups. When using the combined criteria for cure, 10/26 (38\%) macroadenomas were cured in the pretreatment group compared with 6/25 $(24 \%)$ in the direct surgery group 1 year postoperatively $(P=0.27)$, and 9/22 (41\%) vs 6/22 (27\%) macroadenomas, respectively, 5 years postoperatively $(P=0.34)$. In the meta-analysis, $16 / 45(36 \%)$ macroadenomas were cured using combined criteria in the pretreatment group vs $8 / 45(18 \%)$ in the direct surgery group after $6-12$ months $(P=0.06)$, and $15 / 41(37 \%)$ vs $8 / 42(19 \%)$, respectively, in the long-term $(P=0.08)$.

Conclusion: This study does not prove a beneficial effect of SSA pre-surgical treatment, but in the meta-analysis a trend towards significance can be claimed. A potential favourable, clinically relevant response cannot be excluded.

\section{Introduction}

Acromegaly causes a variety of different clinical symptoms and signs, reflecting the increased morbidity for these patients. In addition, active acromegaly is associated with increased mortality, correlated with elevated growth hormone (GH) (1) and insulin-like growth factor 1 (IGF1) levels $(2,3)$. Therefore, effective treatment of acromegaly is important.
(C) 2014 European Society of Endocrinology Printed in Great Britain
Transsphenoidal pituitary surgery to remove the somatotroph adenoma is considered to be the primary treatment for acromegaly. Despite reports from particularly experienced surgical departments of an overall cure rate as high as 70\% (1), reports from national or regional surveys show markedly lower cure rates of $30-40 \%(4,5,6)$. As expected, the cure rate is 
lower in macroadenomas and particularly in invasive adenomas (1).

Medical treatment of somatotroph adenomas with somatostatin analogues (SSA) causes a decline in hormone levels in the majority of patients, although with large individual differences. Moreover, $\sim 2 / 3$ of naïve patients experience significant tumour shrinkage during treatment with the traditional SSAs $(7,8,9,10)$. These effects were the rationale behind the first studies of preoperative treatment with an SSA. A normalisation of GH and IGF1 could lead to decreased anaesthetical complication rate and lower surgical risk, and both softening and shrinkage of tumour to a less invasive adenoma could facilitate tumour removal $(11,12,13)$. We reported the first randomised, controlled study of preoperative octreotide treatment of acromegaly (the POTA study) in 2008, concluding with a better cure rate at the 3 months post-operative evaluation for patients with macroadenomas who had received 6 months presurgical treatment with the SSA octreotide $(10,14)$. Subsequently, three trials have reported similar results $(15,16,17)$. However, at present three of these altogether four studies have reported cure at 3-4 months postoperatively only, and the fourth one (17) demonstrates no significant difference in cure rate in the long-term.

The primary endpoint of the POTA study was cure at 3 months postoperatively. We showed a significant effect of pretreatment for macroadenomas but not for microadenomas, albeit with very few patients in the latter group. According to the study protocol, the patients were followed and evaluated annually until 5 years after surgery. Long-term observational data in these patients are of importance, also because a concern has been raised of a potential lingering effect of preoperative SSA at the very early post-operative evaluation resulting in potential false positive results $(18,19)$.

In this study, we present the results of the postoperative evaluations 1 and 5 years after surgery from the POTA study. There were a small but not negligible number of patients who were lost to follow-up before the final evaluation. To try to avoid a type 2 statistical error, we also performed a meta-analysis on our data for macroadenomas combined with the results of the previously published randomised controlled study with long-term data (17).

\section{Patients and methods}

\section{Patients}

In the period between September 1999 and October 2004, all newly diagnosed patients with acromegaly in Norway were considered for inclusion in the POTA study. All five university hospitals treating acromegaly in Norway participated in the study. Of all 83 patients, 62 (75\%) were included in the study, as previously described in detail (10).

A written informed consent was obtained from each patient. The study was approved by the Regional Committee for Medical and Health Research Ethics and The Norwegian Medicines Agency, and was conducted according to the Declaration of Helsinki II. ClinicalTrials.gov identifier: NCT00521300.

\section{Study design and treatment}

After a baseline evaluation, the included patients were randomised to either direct transsphenoidal surgery (direct surgery group, $n=30$ ) or to 6 months' treatment with octreotide before surgery (pretreatment group, $n=32$ ). The randomisation was carried out separately for each study centre in blocks of four, but with a central allocation with sealed envelopes.

The patients randomised to pre-surgical octreotide treatment received s.c. octreotide $50 \mu \mathrm{g}$ t.i.d. the first week and then $100 \mu \mathrm{g}$ t.i.d. the second week. From the third week, the patients received octreotide LAR $20 \mathrm{mg}$ i.m. every 28 th day until surgery after $\sim 6$ months. All patients in the pretreatment group underwent surgery with therapeutic levels of octreotide. The clinical effects of octreotide pretreatment have been described previously (10).

All patients were evaluated 3 months postoperatively (14). After this evaluation, the patients could receive additional treatment (medical, re-surgery or radiation) if considered necessary by the endocrinologist at each study centre. No guidelines for the treatment 3 months postoperatively and onwards were stated in the protocol. According to the protocol, the patients were then evaluated at 1 year and annually until 5 years after the initial surgery. One of the patients with microadenoma in the pretreatment group was not operated due to the patient's request based on excellent biochemical and tumour response, and was excluded from the present analyses. Two patients died before the 5-year evaluation, and available data were insufficient for any classification of cure at 5-year post-operative evaluation in an additional three patients, all having macroadenomas. For three patients, no hormone data were available 5 years postoperatively, but since they had received additional postoperative treatment they could be classified as not cured. In total, classification of cure status at 5 years postoperatively was available in 56 patients (46 macroadenomas). 


\section{Investigations}

A central retrospective evaluation of the magnetic resonance imaging scan at inclusion classified each tumour as a macroadenoma (largest diameter $\geq 10 \mathrm{~mm}$ ) or a microadenoma (10).

Fasting serum samples were drawn to analyse IGF1 levels locally, both at 1 and 5 years following initial surgery. At 1 year postoperatively, additional serum was stored at $-70^{\circ} \mathrm{C}$, and IGF1 level was later measured centrally in one run using an ELISA Kit (R\&D Systems, Minneapolis, MN, USA), as described previously (14). In five patients, frozen serum was missing, and the locally measured IGF1 level with the local reference value was used instead. At 5-year post-operative evaluation, serum IGF1 level was measured consecutively by routine methods at each local hospital laboratory. However, except for the two earliest analyses from one of the laboratories where IGF1 levels were measured by a kit from Nichols Institute (Nijmegen, the Netherlands), all laboratories $(n=4)$ used Siemens Immulite (Erlangen, Germany).

An oral glucose tolerance test (OGTT) was performed in all patients 1 year postoperatively, and in 46 patients 5 years postoperatively. GH measurements were performed consecutively at the local hospital laboratories.

\section{Meta-analysis}

Shen et al. (17) present 6 months' follow-up results and long-term results after a mean of 26.6-28.8 months. In this study, 11 patients received gamma-knife therapy about 6 months postoperatively. These patients were all classified as non-cured at the subsequent evaluations (personal communication, Prof. Zhao). We performed one metaanalysis on these 6-months follow-up results combined with our 1-year post-operative results, and another metaanalysis on the last follow-up data from Shen et al. combined with our 5-year post-operative data.

Table 1 Additional treatment for acromegaly until 1 year postoperatively.

\begin{tabular}{lcccc}
\hline & $\begin{array}{c}\text { Preoperative SMS } \\
(n=31)\end{array}$ & & $\begin{array}{c}\text { Direct surgery } \\
(n=30)\end{array}$ \\
\cline { 1 - 1 } Medical therapy $(n)$ & & 8 & 5 \\
SMS & 1 & 3 \\
Pegvisomant & 1 & 1 \\
SMS + cabergoline & 1 & 1 \\
Radiation therapy $(n)$ & 1 & 4 \\
Repeated surgery $(n)$ & 8 & 2 \\
Total $(n)$ & & \\
\hline
\end{tabular}

\section{Definition of cure}

A patient was considered cured when IGF1 level was equal to or below upper limit of normal (ULN) and GH nadir was $\leq 2 \mathrm{mU} / \mathrm{l}$ during an $75 \mathrm{~g}$ OGTT, without having received any treatment for acromegaly after the initial surgery. Results are also presented for cure defined only by IGF1 level equal to or lower than ULN.

\section{Statistical analyses}

The statistical analyses were performed using SPSS Statistics, version 20.0 (IBM Corp.), except for the metaanalysis and forest plots, which were performed using Review Manager 5.2 (The Nordic Cochrane Centre, Copenhagen, Denmark). The $\chi^{2}$ test was used for categorical variables except for analyses within the microadenoma group where Fisher's exact test was used. $P$ values $<0.05$ were considered significant. No adjustment was made for multiple testing.

\section{Results}

\section{Post-operative acromegaly treatment}

Before the 1-year post-operative evaluation, 17 patients had received additional, post-operative treatment for acromegaly. Of these, eight patients were in the pretreatment group and nine in the direct surgery group (8/31 vs $9 / 30 ; P=0.72$ ). Details regarding the additional treatment are given in Table 1 .

At the 5-year post-operative evaluation, 30/59 patients had received additional treatment, 15 in each treatment group.

\section{Cure at $\mathbf{1}$ year postoperatively}

For cure defined by an IGF1 level $\leq$ ULN, 12/31 (39\%) patients in the pretreatment group and 11/30 (37\%) patients in the direct surgery group were cured without post-operative treatment, $P=0.87$. For macroadenomas, $12 / 26$ (46\%) patients in the pretreatment group vs $8 / 25$ (32\%) patients in the direct surgery group were cured, $P=0.30$. The number of microadenomas was low; details are given in Table 2 .

For cure defined by the combined criteria of IGF $1 \leq$ ULN and a nadir GH during OGTT $\leq 2 \mathrm{mU} / \mathrm{l}$, the cure rate was lower. In the pretreatment group, 10/31 $(32 \%)$ were cured compared with 8/30 (27\%) in the direct surgery group, $P=0.63$. Among the macroadenomas, 
Table 2 Cure at 1 year defined by IGF1 $\leq$ ULN and nadir GH $\leq 2 \mathrm{mU} /$ during OGTT at one year post-operative, without having received any post-operative treatment for acromegaly. Fisher's exact test two-sided used for microadenomas, Pearson Chi-Square used for macroadenomas and total group.

\begin{tabular}{|c|c|c|c|c|c|c|c|c|c|c|}
\hline \multirow[b]{2}{*}{ Treatment group } & \multicolumn{5}{|c|}{ According to IGF1 } & \multicolumn{5}{|c|}{ According to IGF1 and GH } \\
\hline & No. & Cured & Not cured & Cure $\%$ & $P$ value & No. & Cured & Not cured & Cure $\%$ & $P$ value \\
\hline \multicolumn{11}{|l|}{ All } \\
\hline Pretreatment & 31 & 12 & 19 & 39 & 0.87 & 31 & 10 & 21 & 32 & 0.63 \\
\hline Direct surgery & 30 & 11 & 19 & 37 & & 30 & 8 & 22 & 27 & \\
\hline \multicolumn{11}{|l|}{ Microadenomas } \\
\hline Pretreatment & 5 & 0 & 5 & 0 & 0.17 & 5 & 0 & 5 & 0 & 0.44 \\
\hline Direct surgery & 5 & 3 & 2 & 60 & & 5 & 2 & 3 & 40 & \\
\hline \multicolumn{11}{|l|}{ Macroadenomas } \\
\hline Pretreatment & 26 & 12 & 14 & 46 & 0.30 & 26 & 10 & 16 & 38 & 0.27 \\
\hline Direct surgery & 25 & 8 & 17 & 32 & & 25 & 6 & 19 & 24 & \\
\hline
\end{tabular}

$10 / 26(38 \%)$ in the pretreatment group and $6 / 25(24 \%)$ in the direct surgery group were cured, $P=0.27$. Details for the microadenomas are given in Table 2 .

\section{Cure at $\mathbf{5}$ years postoperatively}

Cure defined as IGF1 level $\leq \mathrm{ULN}$ was achieved without any additional post-operative treatment in 12/28 (43\%) patients in the pretreatment group and 11/28 (39\%) in the direct surgery group, $P=0.79$. For macroadenomas, $10 / 23$ (43\%) were cured in the pretreatment group vs $8 / 23$ (35\%) in the direct surgery group, $P=0.55$. Details are given in Table 3 .

For cure defined by both IGF1 and nadir GH levels, $11 / 27$ patients (41\%) were cured in the pretreatment group and $8 / 26$ (31\%) in the direct surgery group, $P=0.45$. Among patients with macroadenomas, 9/22 (41\%) patients were cured in the pretreatment group vs $6 / 22$ patients $(27 \%)$ in the direct surgery group, $P=0.34$. For microadenomas, see Table 3.

\section{Meta-analysis}

When pooling our results from the 1 year post-operative evaluation with 6 months' post-operative data from Shen et al. (17) and using only IGF1 as cure criterion, the number of cured macroadenomas after pretreatment was 20/45 (44\%) for the pretreatment group vs 12/45 (27\%) for the direct surgery group, $P=0.08$. The meta-analysis is visualised in a forest plot, Fig. $1 \mathrm{~A}$.

Accordingly, for cure defined by the combined IGF1 and GH criterion, 16/45 (36\%) patients with macroadenomas were cured in the pretreatment group vs $8 / 45$ (18\%) in the direct surgery group, $P=0.06$, Fig. $1 \mathrm{~B}$.

When our 5-year post-operative data on macroadenomas were pooled with data from the last follow-up in the study by Shen et al., and using only IGF1 as criterion for cure, the number of cured macroadenomas was $17 / 42$ (40\%) in the pretreatment group and 12/43 (28\%) in the direct surgery group, $P=0.23$, Fig. $1 \mathrm{C}$. Using the combined cure criteria, 15/41 (37\%) patients with macroadenomas

Table 3 Cure at 5 year defined by IGF1 $\leq$ ULN and nadir $\mathrm{GH} \leq 2 \mathrm{mU} / \mathrm{l}$ during OGTT at five years post-operative, without having received post-operative treatment for acromegaly. Fisher's exact test two-sided used for microadenomas, Pearson Chi-Square used for macroadenomas and total group.

\begin{tabular}{|c|c|c|c|c|c|c|c|c|c|c|}
\hline \multirow[b]{2}{*}{ Treatment group } & \multicolumn{5}{|c|}{ According to IGF1 } & \multicolumn{5}{|c|}{ According to IGF1 and GH } \\
\hline & No. & Cured & Not cured & Cure \% & $P$ value & No. & Cured & Not cured & Cure \% & $P$ value \\
\hline \multicolumn{11}{|l|}{ All } \\
\hline Pretreatment & 28 & 12 & 16 & 43 & 0.79 & 27 & 11 & 16 & 41 & 0.45 \\
\hline $\begin{array}{l}\text { Direct surgery } \\
\text { Microadenomas }\end{array}$ & 28 & 11 & 17 & 39 & & 26 & 8 & 18 & 31 & \\
\hline Pretreatment & 5 & 2 & 3 & 40 & 1.0 & 5 & 2 & 3 & 40 & 1.0 \\
\hline $\begin{array}{l}\text { Direct surgery } \\
\text { Macroadenomas }\end{array}$ & 5 & 3 & 2 & 60 & & 4 & 2 & 2 & 50 & \\
\hline Pretreatment & 23 & 10 & 13 & 43 & 0.55 & 22 & 9 & 13 & 41 & 0.34 \\
\hline Direct surgery & 23 & 8 & 15 & 34 & & 22 & 6 & 16 & 27 & \\
\hline
\end{tabular}

www.eje-online.org 


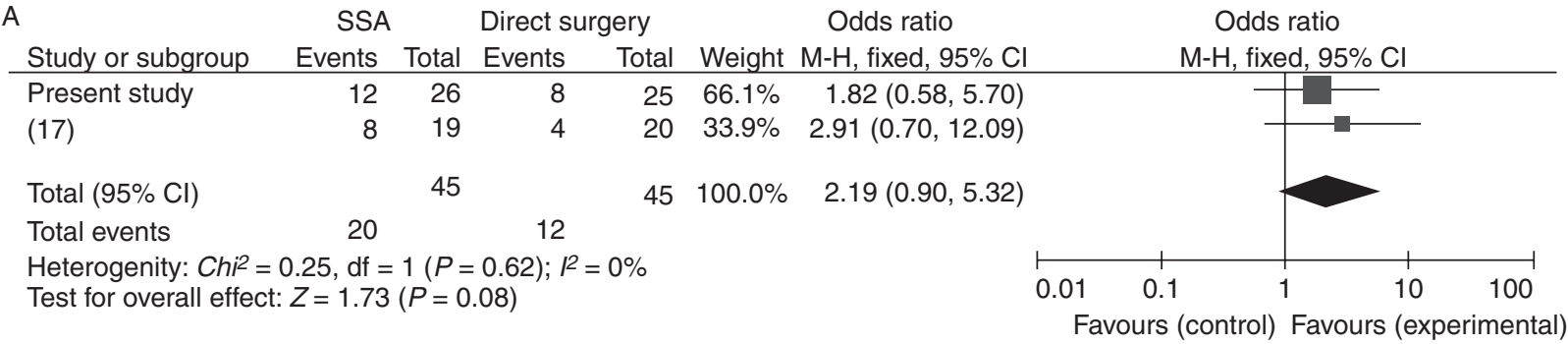

B SSA Direct surgery $\quad$ Odds ratio $\quad$ Odds ratio

Study or subgroup Events Total Events Total Weight $\mathrm{M}-\mathrm{H}$, fixed, $95 \% \mathrm{Cl} \quad \mathrm{M}-\mathrm{H}$, fixed, $95 \% \mathrm{Cl}$

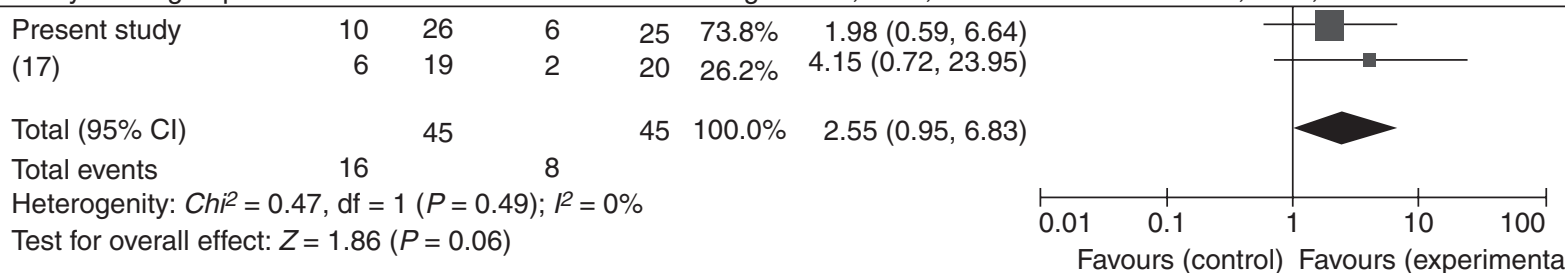

C SSA Direct surgery $\quad$ Odds ratio Odds ratio

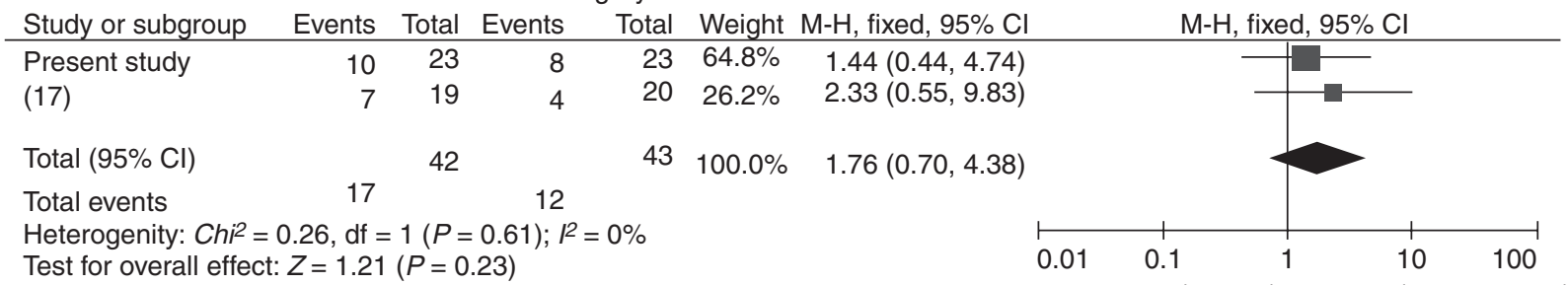

Test for overall effect: $Z=1.21(P=0.23)$

Favours (control) Favours (experimental)

D SSA $\quad$ Direct surgery $\quad$ Risk ratio $\quad$ Odds ratio

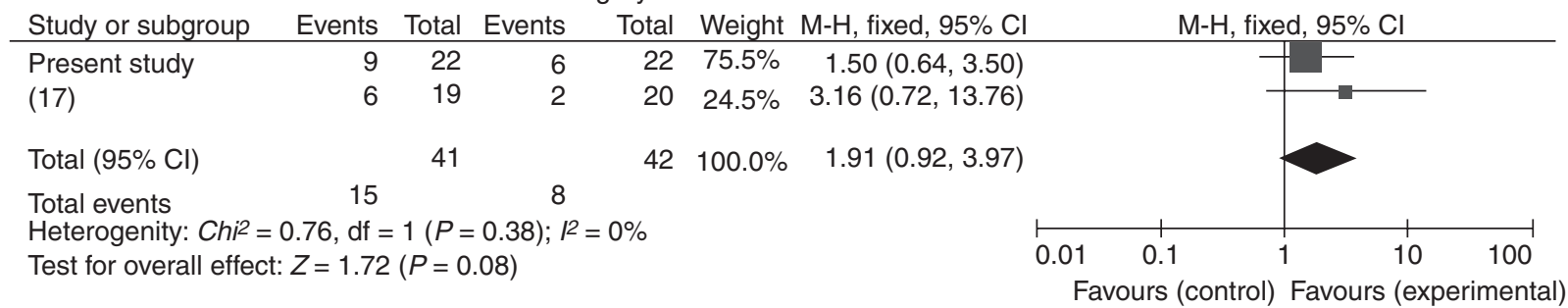

\section{Figure 1}

Meta-analyses. A and B, Meta-analyses of cure at 6-12 months post-operative evaluation; A, Cure defined by IGF1 $\leq$ ULN; B, Cure defined by IGF1 $\leq$ ULN and nadir GH during OGTT $\leq 2 \mathrm{mU} / \mathrm{l} ; \mathrm{C}$ and $\mathrm{D}$, Meta-analyses of cure at long-term post-operative evaluation, 5 years (present study) and last follow-up (17). C, Cure defined by IGF1 $\leq$ ULN. D, Cure defined by IGF1 $\leq$ ULN and nadir GH during $\mathrm{OGTT} \leq 2 \mathrm{mU} / \mathrm{l}$. Full colour version of this figure available via http://dx.doi.org/10.1530/EJE-14-0249.

were cured in the pretreatment group vs $8 / 42$ (19\%) in the direct surgery group, $P=0.08$, Fig. $1 \mathrm{D}$.

\section{Discussion}

This randomised study of preoperative SSA treatment in acromegaly is the second study to present randomised long-term cure data. No significant impact of preoperative treatment of macroadenomas is found at 1 and 5 years postoperatively, despite the previously published benefit for macroadenomas at the 3-month post-operative evaluation. However, when pooling the data with the other long-term study, a trend towards benefit of pretreatment can be claimed. For macroadenomas, twice as many patients were cured without additional treatment 6-12 months after the initial surgery in the pretreatment 
group (36\%) vs the direct surgery group (18\%). The results in our study were less discriminate, but with the same tendency as in the Chinese study. A possible explanation is that Shen et al. only included invasive macroadenomas and demonstrate a correlation of cure to shrinkage to a less invasive adenoma during pretreatment. Another possible bias of the meta-analysis is that the studies were heterogeneous with respect to the duration of octreotide treatment preoperatively, and that the 6 -month postoperative evaluation in Shen et al.'s study may not be entirely comparable with the 12-month evaluation in our study. Nevertheless, data are available for only 90 patients altogether, with a potential risk of a type 2 statistical error. The 5-year post-operative results are also comparable, representing a $50 \%$ increase in absolute numbers, but not statistically significant. When presenting the data combined with the long-term results reported by Shen et al. (17), the result is very similar to the metaanalysis 6-12 months postoperatively, with a trend for increased cure of macroadenomas by pretreatment representing a doubled cure rate in the pretreated group.

Particularly octreotide but also lanreotide has been proven to have a long biological half-life when given in slow-release formulas, and can be detected in circulation many weeks after injection $(20,21)$. A phase I study demonstrated that the concentration of octreotide LAR decreases slowly to immeasurable values in the first 11 weeks after a single injection (22). A clinical study of SSA withdrawal has demonstrated suppressed hormone levels for several months in some patients (23). A potential hangover effect of SSA at the 3-4 months' post-operative evaluation causing false results is therefore possible, necessitating analysis of long-term cure data. This POTA study was planned with yearly assessments from 1 to 5 years postoperatively, and all patients underwent the first year post-operative control.

Owing to lack of cure after the initial surgery, several patients received post-operative additional treatment. The proportion of patients receiving adjuvant treatment was equal in both groups. However, the study protocol did not standardise the indication for, or the selection of, adjuvant therapy. Within the first year both gamma-knife therapy and medical treatment were given, as well as renewed surgery in a few patients. Medical treatment was chosen in all pretreated patients (8/8), compared with 5/9 patients in the direct surgery group. Nevertheless, all patients receiving additional treatment were considered as not cured despite hormone levels and this would therefore does not affect the cure rate in the long-term. However, it is the hormone status in the long term that is clinically relevant and important for each patient. Among the 40 patients considered as not cured after IGF1 3 months postoperatively (14), less than half of the patients received additional acromegaly treatment at 1 year postoperatively. This demonstrates undertreatment in several patients, but can probably also reflect a discrepancy between the locally measured IGF1 level and the central measurement performed both at 3 and 12 months postoperatively. This discrepancy may also explain the lower proportion of cured patients at 1 year compared with 5-year postoperatively, when only locally measured IGF1 levels were available.

In this study, all patients with newly diagnosed acromegaly were considered for inclusion. Therefore, in contrast to the other published studies on preoperative SSA treatment, also microadenomas were included. The proportion of microadenomas was low, but fortunately, the randomisation ensured five patients with microadenomas in each treatment group. As discussed in our first paper (14), a possible unfavourable effect of pretreatment could be suspected when considering cure rate at 3 and 12 months following surgery since none of the direct surgery microadenomas were cured. However, at 5-year post-operative evaluation the results were equal in the two groups. Anyway, the groups were too small to draw any conclusions, and additional studies are warranted.

In conclusion, this randomised study of preoperative treatment with SSA in acromegaly did not provide statistical documentation for a beneficial long-term effect. In meta-analyses of a total of 90 and 83 patients evaluated at 6-12 months and later, respectively, approximately twice as many patients were cured among the pretreated patients compared with the direct surgery group. However, there were not significant differences between the cure rates. Therefore, more long-term response studies are required. If future studies show effects comparable to the present results, this would be very important as a doubled surgical cure rate would be a major improvement in the treatment of acromegaly.

\section{Declaration of interest}

The authors declare that there is no conflict of interest that could be perceived as prejudicing the impartiality of the research reported. S L Fougner and S M Carlsen have received lecture fees/travel grants from Ipsen Norway and Novartis Norway.

\section{Funding}

This study was carried out by the POTA study group which is a subgroup of the 'Norwegian Neuroendocrine Interest group' (NNI). The annual 
meetings of $\mathrm{NNI}$, where all authors have participated, are supported by Novartis Norway. The study was also directly supported by Novartis Norway who paid the salary for a part-time study nurse (20\% position), supported the drug used (Sandostatin and Sandostatin LAR) free of charge and carried the expenses when the study nurse checked the raw data at each study site.

\section{References}

1 Jane JA Jr, Starke RM, Elzoghby MA, Reames DL, Payne SC, Thorner MO, Marshall JC, Laws ER Jr \& Vance ML. Endoscopic transsphenoidal surgery for acromegaly: remission using modern criteria, complications, and predictors of outcome. Journal of Clinical Endocrinology and Metabolism 201196 2732-2740. (doi:10.1210/jc.2011-0554)

2 Dekkers OM, Biermasz NR, Pereira AM, Romijn JA \& Vandenbroucke JP. Mortality in acromegaly: a metaanalysis. Journal of Clinical Endocrinology and Metabolism 200893 61-67. (doi:10.1210/jc.2007-1191)

3 Holdaway IM, Bolland MJ \& Gamble GD. A meta-analysis of the effect of lowering serum levels of GH and IGF-I on mortality in acromegaly. European Journal of Endocrinology 2008159 89-95. (doi:10.1530/EJE-080267)

4 Bates PR, Carson MN, Trainer PJ, Wass JA \& Group UKNARS. Wide variation in surgical outcomes for acromegaly in the UK. Clinical Endocrinology 200868 136-142. (doi:10.1111/j.1365-2265.2007. 03012.x)

5 Bex M, Abs R, T'Sjoen G, Mockel J, Velkeniers B, Muermans K \& Maiter D. AcroBel - the Belgian registry on acromegaly: a survey of the 'real-life' outcome in 418 acromegalic subjects. European Journal of Endocrinology 2007157 399-409. (doi:10.1530/EJE-07-0358)

6 Mestron A, Webb SM, Astorga R, Benito P, Catala M, Gaztambide S, Gomez JM, Halperin I, Lucas-Morante T, Moreno B et al. Epidemiology, clinical characteristics, outcome, morbidity and mortality in acromegaly based on the Spanish Acromegaly Registry (Registro Espanol de Acromegalia, REA). European Journal of Endocrinology 2004151 439-446. (doi:10.1530/eje.0.1510439)

7 Annamalai AK, Webb A, Kandasamy N, Elkhawad M, Moir S, Khan F, Maki-Petaja K, Gayton EL, Strey CH, O'Toole S et al. A comprehensive study of clinical, biochemical, radiological, vascular, cardiac, and sleep parameters in an unselected cohort of patients with acromegaly undergoing presurgical somatostatin receptor ligand therapy. Journal of Clinical Endocrinology and Metabolism 201398 1040-1050. (doi:10.1210/ jc.2012-3072)

8 Luque-Ramirez M, Portoles GR, Varela C, Albero R, Halperin I, Moreiro J, Soto A, Casamitjana R \& Spanish Multicentre Group for the Study of Acromegaly. The efficacy of octreotide LAR as firstline therapy for patients with newly diagnosed acromegaly is independent of tumor extension: predictive factors of tumor and biochemical response. Hormone and Metabolic Research 201042 38-44. (doi:10.1055/s-00291239506)

9 Mercado M, Borges F, Bouterfa H, Chang TC, Chervin A, Farrall AJ, Patocs A, Petersenn S, Podoba J, Safari M et al. A prospective, multicentre study to investigate the efficacy, safety and tolerability of octreotide LAR (long-acting repeatable octreotide) in the primary therapy of patients with acromegaly. Clinical Endocrinology 200766 859-868. (doi:10.1111/j.1365-2265.2007.02825.x)

10 Carlsen SM, Svartberg J, Schreiner T, Aanderud S, Johannesen A, Skeie S, Lund-Johansen M, Fougner SL, Bollerslev J \& POTA study group. Six-month preoperative octreotide treatment in unselected, de novo patients with acromegaly: effect on biochemistry, tumour volume, and postoperative cure. Clinical Endocrinology 201174 736-743. (doi:10.1111/j.1365-2265.2011.03982.x)

11 Colao A, Ferone D, Cappabianca P, Del Basso De Caro ML, Marzullo P, Monticelli A, Alfieri A, Merola B, Calì A, de Divitiis E et al. Effect of octreotide pretreatment on surgical outcome in acromegaly. Journal of Clinical Endocrinology and Metabolism 199782 3308-3314. (doi:10.1210/ jcem.82.10.4283)

12 Stevenaert A \& Beckers A. Presurgical octreotide treatment in acromegaly. Acta Endocrinologica 1993129 (Suppl 1) 18-20.

13 Barkan AL, Lloyd RV, Chandler WF, Hatfield MK, Gebarski SS, Kelch RP $\&$ Beitins IZ. Preoperative treatment of acromegaly with long-acting somatostatin analog SMS 201-995: shrinkage of invasive pituitary macroadenomas and improved surgical remission rate. Journal of Clinical Endocrinology and Metabolism 198867 1040-1048. (doi:10.1210/ jcem-67-5-1040)

14 Carlsen SM, Lund-Johansen M, Schreiner T, Aanderud S, Johannesen O, Svartberg J, Cooper JG, Hald JK, Fougner SL, Bollerslev J et al. Preoperative octreotide treatment in newly diagnosed acromegalic patients with macroadenomas increases cure short-term postoperative rates: a prospective, randomized trial. Journal of Clinical Endocrinology and Metabolism 200893 2984-2990. (doi:10.1210/jc. 2008-0315)

15 Li ZQ, Quan Z, Tian H \& Cheng M. Preoperative lanreotide treatment improves outcome in patients with acromegaly resulting from invasive pituitary macroadenoma. Journal of International Medical Research 2012 40 517-524. (doi:10.1177/147323001204000213)

16 Mao ZG, Zhu YH, Tang HL, Wang DY, Zhou J, He DS, Lan H, Luo BN \& Wang HJ. Preoperative lanreotide treatment in acromegalic patients with macroadenomas increases short-term postoperative cure rates: a prospective, randomised trial. European Journal of Endocrinology 2010 162 661-666. (doi:10.1530/EJE-09-0908)

17 Shen M, Shou X, Wang Y, Zhang Z, Wu J, Mao Y, Li S \& Zhao Y. Effect of presurgical long-acting octreotide treatment in acromegaly patients with invasive pituitary macroadenomas: a prospective randomized study. Endocrine Journal 201057 1035-1044. (doi:10.1507/endocrj. K10E-203)

18 Beckers A. Does preoperative somatostatin analog treatment improve surgical cure rates in acromegaly? A new look at an old question. Journal of Clinical Endocrinology and Metabolism 200893 2975-2977. (doi:10.1210/jc.2008-1351)

19 Pita-Gutierrez F, Pertega-Diaz S, Pita-Fernandez S, Pena L, Lugo G, Sangiao-Alvarellos S \& Cordido F. Place of preoperative treatment of acromegaly with somatostatin analog on surgical outcome: a systematic review and meta-analysis. PLoS ONE 20138 e61523. (doi:10.1371/ journal.pone.0061523)

$20 \mathrm{Hu}$ M \& Tomlinson B. Pharmacokinetic evaluation of lanreotide. Expert Opinion on Drug Metabolism \& Toxicology 20106 1301-1312. (doi:10.1517/17425255.2010.513700)

21 Petersen H, Bizec JC, Schuetz H \& Delporte ML. Pharmacokinetic and technical comparison of Sandostatin(R) LAR(R) and other formulations of long-acting octreotide. BMC Research Notes 20114 344. (doi:10.1186/ 1756-0500-4-344)

22 Astruc B, Marbach P, Bouterfa H, Denot C, Safari M, Vitaliti A \& Sheppard M. Long-acting octreotide and prolonged-release lanreotide formulations have different pharmacokinetic profiles. Journal of Clinical Pharmacology 200545 836-844. (doi:10.1177/0091270005277936)

23 Lorcy Y, Dejager S, Chanson P \& French Octreotide LARG. Time course of GH and IGF-1 levels following withdrawal of long-acting octreotide in acromegaly. Pituitary 20003 193-197. (doi:10.1023/ A:1011416112730)

Received 28 March 2014

Revised version received 22 May 2014

Accepted 27 May 2014 